Rev. Int. Contam. Ambie. 35 (4) 931-943, 2019

DOI: 10.20937/RICA.2019.35.04.12

\title{
REMOCIÓN DE TENSOACTIVOS Y COLIFORMES EN AGUAS RESIDUALES DOMÉSTICAS MEDIANTE PROCESOS FENTON
}

\author{
Reduction of surfactants and coliforms in domestic wastewater by Fenton processes
}

\begin{abstract}
Leandro Darío MORILLO SEMANATE*, David Alejandro NARANJO TOVAR, Jady PÉREZ, William Estuardo VILLACIS OÑATE, Paul VARGAS JENTZSCH y Florinella MUÑOZ BISESTI
\end{abstract}

Departamento de Ciencias Nucleares, Facultad de Ingeniería Química y Agroindustria, Escuela Politécnica Nacional, Ladrón de Guevara E11-253, 170525 Quito, Ecuador

*Autor para correspondencia: leandro.morillo@epn.edu.ec

(Recibido: febrero 2018; aceptado: febrero 2019)

Palabras clave: procesos de oxidación avanzada, remediación ambiental, sustancias activas al azul de metileno, Escherichia coli, ácido cítrico

\section{RESUMEN}

En varios países en desarrollo, el agua residual doméstica es una de las mayores fuentes de contaminación de cuerpos de agua, y los procesos de oxidación avanzada aparecen en la actualidad como una solución promisoria a dicha problemática. En este trabajo se probaron los procesos Fenton convencional y modificado con el agente quelante ácido cítrico para la remoción de tensoactivos y la inactivación de coliformes fecales del efluente de una planta de tratamiento de aguas residuales domésticas localizada en la ciudad de Quito, Ecuador. Se encontraron las mejores condiciones (requerimiento de agitación, concentración de $\mathrm{H}_{2} \mathrm{O}_{2}$ y relación molar $\mathrm{Fe}^{2+}: \mathrm{H}_{2} \mathrm{O}_{2}$ ) para el proceso Fenton convencional. Estas condiciones fueron aplicadas en el Fenton modificado con ácido cítrico. Para este proceso, se probaron dos relaciones molares $\mathrm{Fe}^{2+}$ :citrato $(1: 1$ y $1: 1.25)$ a cuatro valores de $\mathrm{pH}(2.80,4.50,6.00$ y 7.84). Los mejores resultados se alcanzaron al emplear una concentración de $\mathrm{H}_{2} \mathrm{O}_{2}$ de $56.33 \mathrm{mM}$, una relación molar $1: 1: 9$ de $\mathrm{Fe}^{2+}$ :citrato: $\mathrm{H}_{2} \mathrm{O}_{2}$, agitación continua y $\mathrm{pH}$ 6. La aplicación de dichas condiciones permitió una remoción completa de coliformes fecales, una reducción de tensoactivos del 97 \% y la disminución de la demanda química de oxígeno en $89 \%$ en 15 min de tratamiento. Las aguas tratadas resultantes cumplieron con los estándares estipulados en la legislación ecuatoriana para descargas a un cuerpo de agua dulce. Este trabajo provee datos valiosos de la efectividad de los procesos Fenton y los postula como solución potencial para la problemática que implica el tratamiento de aguas residuales domésticas.

Key words: advanced oxidation processes, environmental remediation, methylene blue active substances, Escherichia coli, citric acid

\begin{abstract}
In many developing countries, domestic wastewater is one of the major pollution sources to water bodies, and advanced oxidation processes appear nowadays as a promising solution to this problem. In this work, the conventional Fenton process, and the chelatemodified Fenton process with citric acid, were tested for surfactants and fecal coliforms removal from an effluent of a domestic wastewater treatment plant located in Quito city,
\end{abstract}


Ecuador. The best conditions (agitation requirements, $\mathrm{H}_{2} \mathrm{O}_{2}$ concentration and $\mathrm{Fe}^{2+}: \mathrm{H}_{2} \mathrm{O}_{2}$ molar ratio) for the conventional Fenton process were found. These conditions were applied to the chelate-modified Fenton process with citric acid. For this process, two $\mathrm{Fe}^{2+}$ :citrate molar ratios $(1: 1$ and $1: 1.25)$ were tested at four $\mathrm{pH}$ values $(2.80,4.50,6.00$, and 7.84). The best results were achieved with a $\mathrm{H}_{2} \mathrm{O}_{2}$ concentration of $56.33 \mathrm{mM}$, a $\mathrm{Fe}^{2+}$ : citrate: $\mathrm{H}_{2} \mathrm{O}_{2}$ molar ratio of $1: 1: 9$, continuous stirring and a $\mathrm{pH}$ value of 6 . The application of these conditions allowed a complete removal of fecal coliforms, a $97 \%$ reduction of surfactants and an $89 \%$ decrease in chemical oxygen demand in 15 min of treatment. The resulting treated waters complied with water quality standards for discharge to water bodies according to the Ecuadorian legislation. This work provides valuable data on the effectiveness of these treatments, thus presenting them as potential solutions for the problematic of domestic wastewater treatment.

\section{INTRODUCCIÓN}

En varios países en desarrollo, el agua residual doméstica es una de las fuentes principales de contaminación de los recursos hídricos. La descarga de estos efluentes a un cuerpo de agua puede provocar una severa alteración de los parámetros de calidad del agua, la mortandad de organismos que en él viven y enfermedades en personas que consuman el agua (Ramin et al. 2012, Voloshenko-Rossin et al. 2015, Yaya-Beas et al. 2016).

Las opciones más sustentables para el tratamiento de efluentes domésticos en países en desarrollo probablemente sean los procesos biológicos anaerobios y las lagunas fotosintéticas (lagunas facultativas y de maduración). Por lo general, los procesos mencionados son usados en serie, es decir, primero se trata el efluente de manera anaerobia y luego fotosintéticamente (Mara 2003, Ramin et al. 2012, Yaya-Beas et al. 2016). Al finalizar el tratamiento, el agua puede ser descargada a un cuerpo de agua receptor o aprovechado para riego de ciertos cultivos (Mara 2003, Heinze et al. 2014, alJassim et al. 2015). Sin embargo, la mayor limitación de los sistemas mencionados radica en una inadecuada remoción de sustancias como los tensoactivos y la presencia de microorganismos patógenos en las aguas efluentes (Selvakumar et al. 2009, Ramin et al. 2012).

Los tensoactivos son compuestos orgánicos capaces de alterar significativamente la tensión superficial o interfacial de un sistema; interfieren en el intercambio de gas en los cuerpos de agua y alteran el sistema hormonal de organismos acuáticos (Ivanković y Hrenović 2010, Lee y Saylor 2010). La gran variedad de productos que tienen en sus formulaciones tensoactivos, entre los que se cuentan fármacos, detergentes, pesticidas, cosméticos y artículos de limpieza personal, entre muchos otros (Rosen y Kunjappu 2012), hacen que estos contaminantes fácilmente lleguen a las aguas residuales domésticas.
Además, la aplicación de sistemas biológicos involucra, generalmente, la necesidad de algún proceso adicional de remoción de microorganismos patógenos (Selvakumar et al. 2009, Ramin et al. 2012). El grado de remoción de microorganismos patógenos se evalúa mediante la presencia de coliformes fecales, debido a que estos microorganismos son indicadores de la calidad sanitaria del agua. Es decir, la detección de coliformes fecales en agua indica, con alta probabilidad, la presencia de patógenos entéricos (Pepper et al. 2014). Es mediante el contenido de coliformes fecales como se evalúa la eficiencia de un tratamiento de aguas residuales en la remoción de microorganismos patógenos $\mathrm{y}$, también, se detecta la contaminación de aguas naturales con aguas residuales domésticas (Ramin et al. 2012, Pepper et al. 2014, al-Jassim et al. 2015).

Ante la problemática descrita surge la necesidad de proponer un tratamiento complementario. En la actualidad, los procesos de oxidación avanzada (POA) aparecen como una opción interesante debido a su principal característica, que es la generación de especies altamente oxidantes denominadas radicales hidroxilo $\left({ }^{\circ} \mathrm{OH}\right)$, que tienen un potencial redox de $2.73 \mathrm{~V}$ vs. SHE y reaccionan de manera no selectiva con los compuestos orgánicos e inorgánicos (Gogate y Pandit 2004, Pignatello et al. 2006).

Entre los POA se encuentra el proceso Fenton tradicional, en el cual los radicales son producidos por la reacción del peróxido de hidrógeno $\left(\mathrm{H}_{2} \mathrm{O}_{2}\right)$ con iones ferrosos $\left(\mathrm{Fe}^{2+}\right)$ dentro de un rango limitado de $\mathrm{pH}$ (Pignatello et al. 2006). A pH $<2.50$ se reduce la formación de $\mathrm{HO}^{\circ}$ porque se generan especies como $\left(\mathrm{Fe}[\mathrm{II}]\left[\mathrm{H}_{2} \mathrm{O}\right]\right)^{2+}$ que reaccionan a una menor tasa con el $\mathrm{H}_{2} \mathrm{O}_{2}$. A pH $>4.00$ disminuye la solubilidad de los iones férricos y se precipitan oxihidróxidos de hierro (Gogate y Pandit 2004); por consiguiente, trabajar a dichos valores de $\mathrm{pH}$ ocasionaría una menor remoción de contaminantes (Kos et al. 2010). 
El mecanismo de reacción del proceso Fenton es complejo y comprende una serie de reacciones en cadena. A continuación, se presentan tres ecuaciones representativas del proceso, en las cuales $\mathrm{Fe}^{2+}$ y $\mathrm{Fe}^{3+}$ representan todas las especies en solución en el respectivo estado de oxidación (Pignatello et al. 2006, Selvakumar et al. 2009):

$\mathrm{Fe}^{2+}+\mathrm{H}_{2} \mathrm{O}_{2} \rightarrow \mathrm{Fe}^{3+}+\mathrm{OH}^{-}+\mathrm{OH}^{\cdot}$

$\mathrm{Fe}^{3+}+\mathrm{H}_{2} \mathrm{O}_{2} \rightarrow \mathrm{Fe}^{2+}+\mathrm{H}^{+}+\mathrm{HO}_{2}^{\cdot}$

$\mathrm{Fe}^{3+}+\mathrm{HO}_{2}^{\cdot} \rightarrow \mathrm{Fe}^{2+}+\mathrm{O}_{2} \mathrm{H}^{+}$

En aguas sintéticas, el proceso Fenton ha permitido una reducción del $63 \%$ de tensoactivos (dodecilsulfato de sodio) en $1 \mathrm{~h}$, al usar $60 \mathrm{mM}$ de $\mathrm{H}_{2} \mathrm{O}_{2}$ y $10 \mathrm{mM}$ de $\mathrm{FeSO}_{4}$ (Bandala et al. 2008). Además, este proceso ha permitido una disminución de tres unidades logarítmicas de Escherichia coli (principal coliforme fecal) en efluentes de una planta avícola, en un lapso de 15 min (Hernández 2012).

Por otro lado, el proceso Fenton modificado con agentes quelantes permite que la oxidación pueda efectuarse en un amplio rango de $\mathrm{pH}$ (Pignatello et al. 2006). Como prueba de ello se han desarrollado tratamientos a $\mathrm{pH}$ neutro con remociones significativas $(>70 \%$ ) de los contaminantes (Xue et al. 2009, De Luca et al. 2014, Isch 2016). Si bien existe una amplia variedad de agentes quelantes para procesos Fenton, el citrato (ácido cítrico) destaca por su efectividad en la remoción de contaminantes persistentes, tal como fue reportado en el pasado (Seol y Javandel 2008, Lewis et al. 2009).

La eliminación de microorganismos de las aguas tratadas es importante para evitar un impacto directo e inmediato en la salud de la población y, además, para evitar la aparición de bacterias resistentes a los antibióticos. Se sabe que existen mecanismos a través de los cuales las bacterias son capaces de transferir, de manera horizontal y vertical, genes de resistencia a los antibióticos (Oromí-Durich 2000). Dado que el uso de antibióticos está generalizado en el mundo entero, la microbiota humana posee bacterias que tienen genes de resistencia a los antibióticos y que podrían ser transferidos a bacterias presentes en aguas naturales, sedimentos y suelos (Baquero et al. 2008). Para evitar la rápida aparición y proliferación de cepas resistentes a los antibióticos que puedan poner en serio riesgo la salud de la población, es necesario evitar el contacto de bacterias de origen humano con bacterias del medio ambiente y el agente crucial de transporte (que debe ser controlado) es el agua (Baquero et al. 2008). En un estudio reciente, se demostró que la resistencia que tienen ciertas bacterias a los antibióticos no las hace resistentes a tratamientos basados en POA (Giannakis et al. 2018), lo cual indica que este tipo de tratamientos puede ser un gran aporte a la lucha mundial contra la resistencia a los antimicrobianos.

Con base en lo expuesto anteriormente, la presente investigación se encaminó a estudiar los procesos Fenton convencional y modificado con el agente quelante ácido cítrico para remover tensoactivos y coliformes en aguas residuales del barrio Chaquibamba perteneciente al Distrito Metropolitano de Quito, Ecuador.

\section{MATERIALES Y MÉTODOS}

\section{Caracterización del efluente pretratado}

En un periodo de ocho semanas se tomaron nueve muestras aleatorias simples del efluente doméstico de una planta de tratamiento de aguas residuales (PTAR), con un proceso biológico anaerobio, localizada en la ciudad de Quito, Ecuador (barrio Chaquibamba). Dichas muestras fueron preservadas según las metodologías APHA 1060 y APHA 9060 (APHA/ AWWA/WEF 2012). El volumen de la muestra aleatoria simple fue de $1 \mathrm{~L}$ y se tomó a la salida del filtro anaerobio de flujo ascendente (FAFA).

En cada una de las muestras simples se efectuaron análisis de los parámetros $\mathrm{pH}$, tensoactivos $\mathrm{y}$ coliformes fecales. La medición del $\mathrm{pH}$ se realizó de acuerdo con el método APHA 4500-H +B. Se utilizó el pH-metro Milwaukee Mi 151 (APHA/AWWA/ WEF 2012).

La concentración de tensoactivos se determinó con el método de sustancias activas al azul de metileno (SAAM, APHA 5540 C). Para ello se realizó una etapa de extracción con cloroformo y azul de metileno, se efectuó un lavado con una solución de fosfato monobásico monohidratado de sodio $\left(\mathrm{NaH}_{2} \mathrm{PO}_{4}\right)$ para eliminar las interferencias y se obtuvo la medición espectrofotométrica a $652 \mathrm{~nm}$ mediante un espectrofotómetro Thermo Scientific Genesys 20 (APHA/AWWA/WEF 2012).

Las coliformes fecales (Escherichia coli) se determinaron mediante placas Petrifilm de $3 \mathrm{M}$ y se realizó la lectura según el método NMKL 147 (NMKL 2015). Para dicho análisis se utilizaron un autoclave All American 25 X, una cámara de flujo laminar Labconco 36000 y una incubadora Memmert B 30. 
Con los valores de los análisis (por duplicado) de $\mathrm{pH}$, tensoactivos (SAAM) y coliformes fecales, se elaboraron cartas de control para establecer la variación de los parámetros mencionados. En función de dichas cartas se estableció el muestreo compuesto requerido. La muestra compuesta a la salida del FAFA fue de $16 \mathrm{~L}$ y fue obtenida por la mezcla de cuatro muestras simples de $4 \mathrm{~L}$ recogidas cada $2 \mathrm{~h}$.

\section{Proceso Fenton convencional. Primera etapa}

Para llevar a cabo el proceso Fenton convencional, se ajustó el valor de $\mathrm{pH}$ del efluente a 2.80 con la adición de $\mathrm{H}_{2} \mathrm{SO}_{4} 1 \mathrm{~N}$. A continuación se adicionó sulfato ferroso heptahidratado ACS $\left(\mathrm{FeSO}_{4} \cdot 7 \mathrm{H}_{2} \mathrm{O}\right)$, se homogeneizó y se inició el tratamiento con la adición de peróxido de hidrógeno $\left(\mathrm{H}_{2} \mathrm{O}_{2}\right) 30 \%$ ACS.

En esta etapa se consideraron dos variables de diseño: $a)$ la relación en masa $(\mathrm{g} / \mathrm{g})$ de tensoactivos: $\mathrm{H}_{2} \mathrm{O}_{2}$ con tres niveles y $b$ ) la agitación con dos niveles, lo que resultó en un diseño experimental $3 \times 2$ factores. La relación en masa de tensoactivos: $\mathrm{H}_{2} \mathrm{O}_{2}$ tomó los valores 1:80, 1:180 y 1:280, mientras las intensidades de agitación con las que se trabajó fueron $130 \mathrm{rpm}$ (Kitis et al. 1999) y 0 rpm (sin agitación continua), para lo cual se usó el equipo de agitación múltiple Phipps \& Bird PB-700. Se efectuaron ensayos sin agitación continua en respuesta al hecho de que en las zonas rurales la disponibilidad de recursos es limitada y el suministro de electricidad podría ser intermitente. Los ensayos sin agitación simularían un escenario extremo en el que el suministro de electricidad hubiera sido suspendido.

Cada prueba se llevó a cabo a temperatura ambiente $\left(17-19{ }^{\circ} \mathrm{C}\right)$, en modalidad de procesamiento por lotes, a un valor de $\mathrm{pH} 2.80$ (Pignatello et al. 2006), en un volumen experimental de $100 \mathrm{~mL}$ y con una relación molar 1:9 de $\mathrm{Fe}^{2+}: \mathrm{H}_{2} \mathrm{O}_{2}$. Las muestras para análisis se tomaron a los 60 min (Bandala et al. 2008).

Para fijar la concentración de $\mathrm{H}_{2} \mathrm{O}_{2}$, la mejor relación tensoactivos: $\mathrm{H}_{2} \mathrm{O}_{2}$ fue aplicada a la concentración más alta de tensoactivos, reportada en las cartas de control, obtenidas en la etapa anterior.

\section{Proceso Fenton convencional. Segunda etapa}

Se complementó el estudio del proceso Fenton convencional mediante la evaluación de la relación molar $\mathrm{Fe}^{2+}: \mathrm{H}_{2} \mathrm{O}_{2}$ en tres niveles: 1:6 (Bandala et al. 2008), 1:9 y 1:16, lo que resultó en un diseño experimental unifactorial. Las alícuotas para los análisis se tomaron a los $15,30,60,90,120$ y 180 min de iniciado el tratamiento Fenton convencional (Bandala et al. 2008, Chitra et al. 2014). Los tiempos mencionados se plantearon al considerar también una necesidad de mayor tiempo de residencia para el tratamiento debido a la naturaleza de la PTAR de Chaquibamba y a los posibles recursos disponibles para la implementación de un tratamiento complementario.

La concentración de $\mathrm{H}_{2} \mathrm{O}_{2}$ y el requerimiento de agitación utilizados, fueron establecidos en la etapa anterior. Se trabajó a pH 2.80 (Pignatello et al. 2006), a temperatura ambiente $\left(17-19^{\circ} \mathrm{C}\right)$, en una unidad experimental de $600 \mathrm{~mL}$ y en modalidad de procesamiento por lotes.

\section{Proceso Fenton modificado con ácido cítrico}

Para llevar a cabo un proceso Fenton modificado fue necesaria la preparación del quelato o complejo citrato- $\mathrm{Fe}^{2+}$. Para ello, se mezclaron volúmenes apropiados de soluciones de ácido cítrico monohidratado $\left(\mathrm{C}_{6} \mathrm{H}_{8} \mathrm{O}_{7} \cdot \mathrm{H}_{2} \mathrm{O}\right)$ y FeSO ${ }_{4} \cdot 7 \mathrm{H}_{2} \mathrm{O}$ con el fin de obtener la relación $\mathrm{Fe}^{2+}$ :citrato requerida. Se ajustó el $\mathrm{pH}$ a 2.80 por adición de una solución de hidróxido de sodio $(\mathrm{NaOH}) 1 \mathrm{~N}$ y se agitó por $30 \mathrm{~min}$. A continuación, para asegurar la formación del complejo, se ajustó el valor de $\mathrm{pH}$ a 4.50 mediante la adición de solución de $\mathrm{NaOH} 1 \mathrm{~N}$ y se agitó por 30 min. Luego se llevó a pH neutro con adición de solución de $\mathrm{NaOH} 1 \mathrm{~N}$ (Lewis et al. 2009, Klamerth et al. 2013). La solución neutralizada del quelato fue añadida a la muestra de agua residual doméstica y se reguló el $\mathrm{pH}$ a los valores requeridos mediante la adición de $\mathrm{H}_{2} \mathrm{SO}_{4} 1 \mathrm{~N}$. Para dar inicio al tratamiento se adicionó $\mathrm{H}_{2} \mathrm{O}_{2} 30 \%$.

En esta etapa se evaluaron dos relaciones molares $\mathrm{Fe}^{2+}$ :citrato a cuatro valores de $\mathrm{pH}$, lo que resultó en un diseño experimental $4 \times 2$ factores. Las relaciones molares evaluadas fueron 1:1 y 1:1.25 (Lewis et al. 2009, De Luca et al. 2014), mientras el pH tomó valores de 2.80, 4.50, 6.00 y 7.84 (pH del efluente) (Gogate y Pandit 2004).

Cada prueba se llevó a cabo con las mejores condiciones (requerimiento de agitación, concentración de $\mathrm{H}_{2} \mathrm{O}_{2}$ y relación molar $\mathrm{Fe}^{2+}: \mathrm{H}_{2} \mathrm{O}_{2}$ ) establecidas para el Fenton tradicional. La experimentación se realizó a temperatura ambiente $\left(17-19{ }^{\circ} \mathrm{C}\right)$, en un volumen experimental de $600 \mathrm{~mL}$ y en modalidad de procesamiento por lotes. Las muestras para análisis se tomaron a $15,30,60,90,120$ y 180 min (Bandala et al. 2008, Chitra et al. 2014).

Los tratamientos expuestos anteriormente se efectuaron por duplicado. Luego de cada ensayo se realizaron análisis de tensoactivos (SAAM) y coliformes fecales, con lo cual se obtuvieron como variables de respuesta sus respectivas concentraciones. Para el estudio estadístico se utilizó el programa STATGRAPHICS Centurion XVI v. 16.1.03, con el cual 
se consiguió el análisis de varianza y los diagramas de medias e interacción.

\section{Demanda química de oxígeno bajo las mejores condiciones de Fenton modificado}

Con la finalidad de conocer el efecto de los radicales hidroxilo sobre otros contaminantes presentes en aguas residuales de origen doméstico, se incluyó la demanda química de oxígeno (DQO) como parámetro de evaluación y se realizó una experimentación por triplicado, en las condiciones establecidas en la etapa anterior.

La caracterización de la muestra compuesta sin tratamiento (inicial) y del efluente tratado (final) se obtuvo mediante las mediciones de DQO, tensoactivos (SAAM) y coliformes fecales. La evaluación se realizó en una segunda muestra compuesta con el fin de comprobar la efectividad de las condiciones propuestas en las etapas anteriores.

La determinación de la DQO se efectuó de conformidad con el método colorimétrico (APHA 5220 D). Se emplearon viales de digestión y un reactor $\mathrm{DQO}$, de $\mathrm{HACH}$. La medición espectrofotométrica se efectuó en un espectrofotómetro HACH DR 2800 (APHA/AWWA/WEF 2012).

Para evitar interferencias debidas al $\mathrm{H}_{2} \mathrm{O}_{2}$ y al $\mathrm{Fe}^{2+}$ en la medición de DQO, se incrementó el pH de la solución hasta un valor de 12.0, se dejó reposar 15 min y luego se redujo a $\mathrm{pH}$ neutro. Al subir el $\mathrm{pH}$ hasta un valor tan alto, el $\mathrm{H}_{2} \mathrm{O}_{2}$ residual del proceso Fenton se descompone y, además, el hierro se precipita como hidróxido (Abbot y Brown 1990, Goi y Trapido 2002).

\section{RESULTADOS Y DISCUSIÓN}

\section{Caracterización del efluente pretratado}

En las figuras 1, 2 y 3 se presentan las cartas de control de los parámetros tensoactivos (SAAM), coliformes fecales y $\mathrm{pH}$, respectivamente. Los datos necesarios para elaborar dichas cartas se obtuvieron de la etapa de muestreo aleatorio del efluente de la PTAR del barrio Chaquibamba.

En las figuras citadas se pueden observar las medias, los límites de alarma superior (LAS) e inferior (LAI), y los límites de control superior (LCS) e inferior (LCI) (APHA/AWWA/WEF 2012). Dichos límites son parámetros estadísticos que permiten conocer la variabilidad de un proceso. Los valores alarma definen el rango fuera del cual existe una desviación aceptable pero con tendencia a un descontrol en el proceso; por otro lado, los límites de control

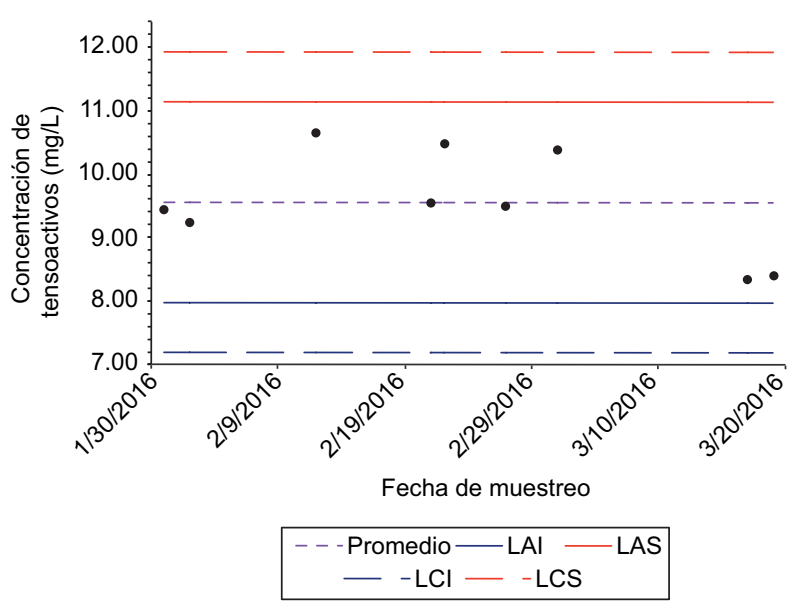

Fig. 1. Carta de control de sustancias activas al azul de metileno del efluente proveniente de la planta de tratamiento de aguas residuales del barrio Chaquibamba, correspondiente a un periodo de ocho semanas entre enero y marzo de 2016. LAI: límite de alarma inferior, LAS: límite de alarma superior, LCI: límite de control inferior, LCS: límite control superior

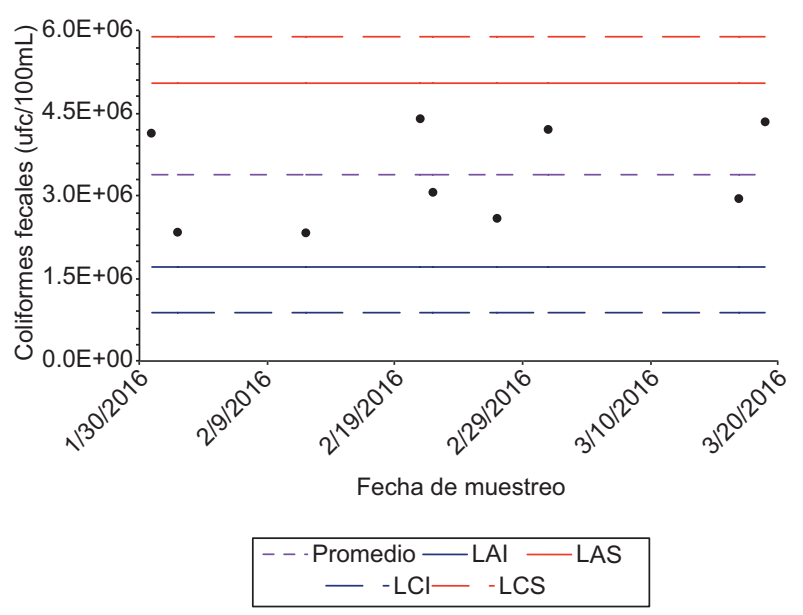

Fig. 2. Carta de control de coliformes fecales del efluente proveniente de la planta de tratamiento de aguas residuales del barrio Chaquibamba, correspondiente a un periodo de ocho semanas entre enero y marzo de 2016. LAI: límite de alarma inferior, LAS: límite de alarma superior, LCI: límite de control inferior, LCS: límite control superior

establecen un rango fuera del cual el proceso necesita corrección puesto que la desviación es inaceptable. En las figuras 1, 2 y 3, se observa que los valores de los parámetros analizados (tensoactivos, coliformes fecales y $\mathrm{pH}$ ) se encontraron dentro del rango establecido por los límites de control, lo que implica que el día de muestreo no influyó en la representatividad de la muestra, razón por la cual los muestreos 


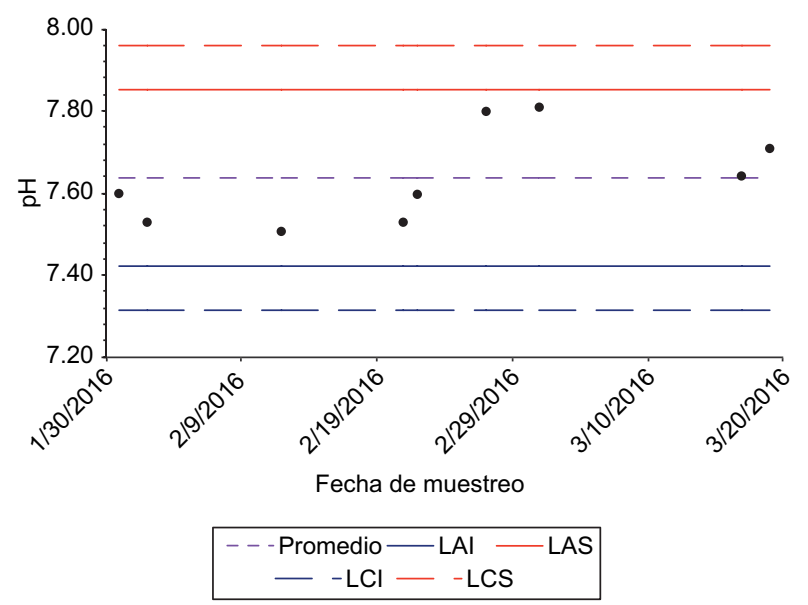

Fig. 3. Carta de control del valor de $\mathrm{pH}$ del efluente proveniente de la planta de tratamiento de aguas residuales del barrio Chaquibamba, correspondiente a un periodo de ocho semanas entre enero y marzo de 2016. LAI: límite de alarma inferior, LAS: límite de alarma superior, LCI: límite de control inferior, LCS: límite control superior

compuestos se llevaron a cabo en días normales de operación (APHA/AWWA/WEF 2012).

Además, las concentraciones de tensoactivos y coliformes fecales obtenidas en esta etapa incumplen con lo establecido en la legislación ecuatoriana para descarga a cuerpos de agua dulce, mientras que el $\mathrm{pH}$ sí se mantiene dentro del rango permitido en dicha normativa (MAE 2015).

El cuadro I indica los valores de los parámetros físicos, químicos y microbiológicos determinados en la muestra compuesta del efluente de estudio y los valores de concentración que no representan un riesgo para la salud, es decir, lo límites máximos permitidos de acuerdo con las leyes ecuatorianas, los cuales son equivalentes a las recomendaciones realizadas por la agencia de protección ambiental de los Estados Unidos (USEPA, por sus siglas en inglés)

CUADRO I. CARACTERIZACIÓN FÍSICA, QUÍMICA Y MICROBIOLÓGICA DEL EFLUENTE DE LA PLANTA DE TRATAMIENTO DE AGUAS RESIDUALES DEL BARRIO CHAQUIBAMBA

\begin{tabular}{lcc}
\hline Parámetro & $\begin{array}{c}\text { Muestra } \\
\text { compuesta }\end{array}$ & $\begin{array}{c}\text { Límite máximo } \\
\text { permisible* }\end{array}$ \\
\hline $\mathrm{pH}$ & 7.84 & $6.00-9.00$ \\
Tensoactivos $(\mathrm{mg} / \mathrm{L})$ & 8.24 & 0.50 \\
Coliformes fecales & $2.8 \mathrm{E}+06$ & $2.0 \mathrm{E}+03$ \\
(UFC/100 mL) & & \\
\hline
\end{tabular}

*MAE 2015, UFC: unidades formadoras de colonias para fuentes de agua destinadas al consumo público (USEPA 1972, MAE 2015). La caracterización de la muestra compuesta denota la deficiencia del tratamiento biológico anaerobio (fosa séptica y FAFA) aplicado en la PTAR de Chaquibamba y reafirma la necesidad de un proceso complementario.

\section{Proceso Fenton convencional. Primera etapa}

En esta etapa se estudiaron la relación en masa $(\mathrm{g} / \mathrm{g})$ tensoactivos: $\mathrm{H}_{2} \mathrm{O}_{2}$ y la influencia de la agitación. Al efectuar el análisis de varianza con un nivel de confianza del $95 \%$, se determinó que ambos factores presentaron efectos significativos durante el tratamiento y que existió una interacción entre los factores en estudio, puesto que los valores $P$ fueron menores a 0.05 .

Como se muestra en la figura 4, se alcanzó una mayor eficacia cuando se trabajó con agitación continua (130 rpm), puesto que la homogeneización y distribución de los contaminantes y de las especies oxidantes, aumentó las probabilidades de contacto entre ellos. Por otro lado, con la relación en masa tensoactivos: $\mathrm{H}_{2} \mathrm{O}_{2}$ de 1:280 se alcanzó una mayor remoción de tensoactivos (SAAM).

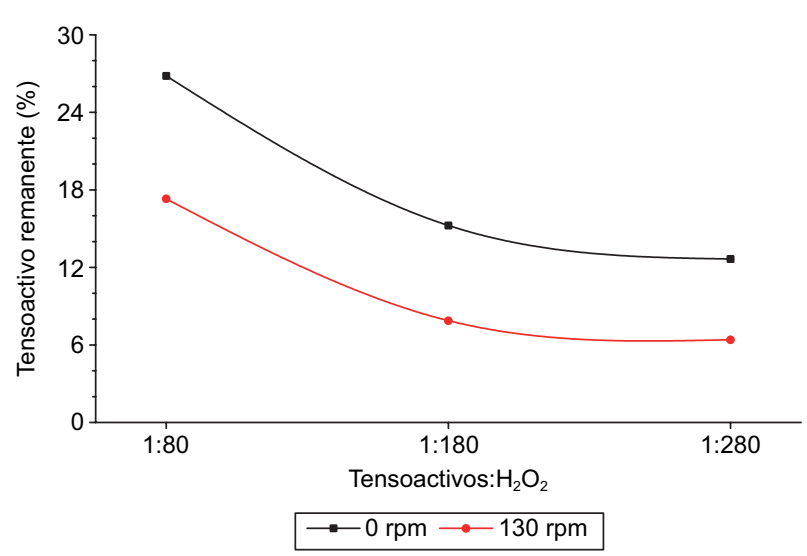

Fig. 4. Variación del porcentaje remanente de sustancias activas al azul de metileno en el efluente de la planta de tratamiento de aguas residuales de Chaquibamba, tratado mediante Fenton tradicional a diferentes relaciones en masa $(\mathrm{g} / \mathrm{g})$ de tensoactivos: $\mathrm{H}_{2} \mathrm{O}_{2}$ con y sin agitación $\left(\mathrm{pH}=2.80\right.$, relación molar $\left.\mathrm{Fe}^{2+}: \mathrm{H}_{2} \mathrm{O}_{2}=1: 9, \mathrm{t}=60 \mathrm{~min}\right)$

Sin embargo del análisis estadístico correspondiente y mediante una gráfica de interacción (Fig. 5), se determinó que no existieron diferencias significativas entre los resultados obtenidos para las relaciones 1:180 y 1:280. Dicha situación se debió a un posible exceso de $\mathrm{H}_{2} \mathrm{O}_{2}$ que actuó como oxidante de los ra- 
dicales hidroxilo (Kos et al. 2010, Babuponnusami y Muthukumar 2014).

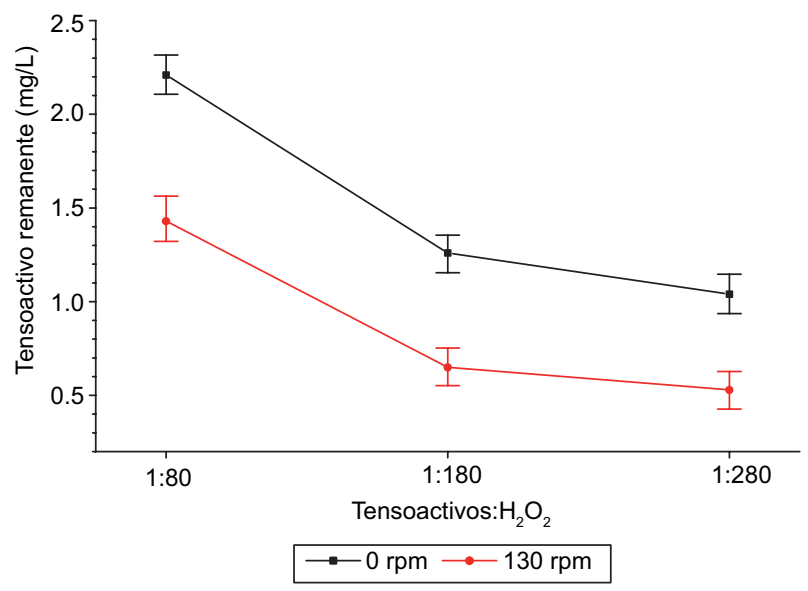

Fig. 5. Gráfica de interacción $(95.0 \%$, prueba de diferencia mínima significativa de Fisher) de las concentraciones de sustancias activas al azul de metileno en el efluente de la planta de tratamiento de aguas residuales de Chaquibamba, tratado mediante un proceso Fenton convencional a diferentes relaciones en masa $(\mathrm{g} / \mathrm{g})$ de tensoactivos: $\mathrm{H}_{2} \mathrm{O}_{2}$ con y sin agitación ( $\mathrm{pH}=2.8$, relación molar $\mathrm{Fe}^{2+}: \mathrm{H}_{2} \mathrm{O}_{2}$ $=1: 9, \mathrm{t}=60 \mathrm{~min})$.

En cuanto a las coliformes fecales, se alcanzó una remoción completa en todos los ensayos realizados en esta etapa. La eficacia de la reacción Fenton para la inactivación de coliformes fecales que se ha demostrado en este trabajo, se debe al posible daño por estrés oxidativo que causan los radicales en la membrana celular de los microorganismos, puesto que la peroxidación lipídica que se desencadena ocasiona una disminución en la fluidez de la membrana y altera sus propiedades (Cabiscol et al. 2000).

Los resultados de inactivación de coliformes fecales guardan relación con el estudio realizado por Selvakumar et al. (2009), en el cual la inactivación completa de Escherichia coli (principal coliforme fecal) en efluentes de una planta de tratamiento de aguas residuales domésticas se consiguió en $0.5 \mathrm{~min}$ a un $\mathrm{pH}$ alrededor de 6 . Otros estudios (Ramin et al. 2012) obtuvieron un $99.92 \%$ de remoción de coliformes fecales, al tratar el efluente de una PTAR de lodos activados en un tiempo de contacto de $30 \mathrm{~min}$. Los resultados de este trabajo, que tienen coherencia con reportes previos, muestran que los procesos Fenton son una gran alternativa para la inactivación de coliformes fecales y, por consiguiente, de distintas bacterias patogénicas de origen fecal. De esta manera se puede asegurar la desinfección de las aguas residuales tratadas (Edberg et al. 2000).

A partir de los resultados obtenidos en esta etapa, se decidió trabajar en las siguientes experimentaciones con agitación de $130 \mathrm{rpm}$ y con la relación en masa $(\mathrm{g} / \mathrm{g})$ tensoactivos: $\mathrm{H}_{2} \mathrm{O}_{2}$ de 1:180 aplicada a la mayor carga de tensoactivos $(10.64 \mathrm{mg} / \mathrm{L})$, cuantificada en las cartas de control, lo que derivó en una concentración de $\mathrm{H}_{2} \mathrm{O}_{2}$ (recalculada) de $1915.20 \mathrm{mg} / \mathrm{L}$, que corresponde a $56.33 \mathrm{mM}$.

\section{Proceso Fenton convencional. Segunda etapa}

En la figura 6 se observa que al utilizar una mayor concentración de $\mathrm{Fe}^{2+}$ se obtuvo una mayor remoción de tensoactivos, dentro de las condiciones aplicadas en este trabajo. En $60 \mathrm{~min}$, el menor porcentaje de remoción ( $89 \%$ ) se obtuvo al utilizar $56.33 \mathrm{mM} \mathrm{H}_{2} \mathrm{O}_{2}$ y $3.52 \mathrm{mM} \mathrm{Fe}^{2+}$, mientras que para el mismo tiempo de reacción, al incrementar la concentración de hierro $\left(56.33 \mathrm{mM} \mathrm{H}_{2} \mathrm{O}_{2}\right.$ y $\left.9.39 \mathrm{mM} \mathrm{Fe}^{2+}\right)$, se consiguió un $95 \%$ de remoción de tensoactivos (SAAM).

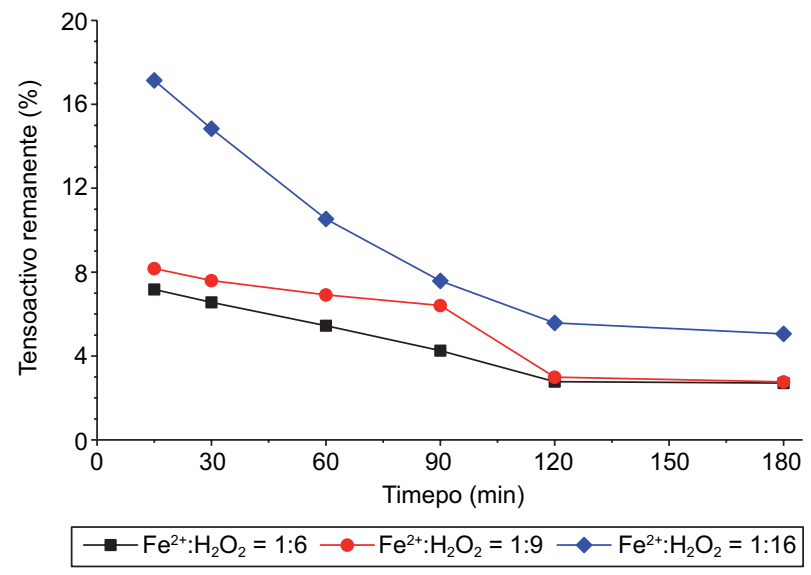

Fig. 6. Variación del porcentaje remanente de sustancias activas al azul de metileno en el efluente de la planta de tratamiento de aguas residuales de Chaquibamba, tratado mediante un proceso Fenton convencional a diferentes relaciones molares $\mathrm{Fe}^{2+}: \mathrm{H}_{2} \mathrm{O}_{2}(\mathrm{pH}=2.80$, $\left.\left[\mathrm{H}_{2} \mathrm{O}_{2}\right]=56.33 \mathrm{mM}\right)$

En otras investigaciones (Bandala et al. 2008) se reportó una reducción del $63 \%$ de tensoactivos en una hora mediante la utilización de $60 \mathrm{mM}$ de $\mathrm{H}_{2} \mathrm{O}_{2}$ y $10 \mathrm{mM}$ de $\mathrm{FeSO}_{4}$ (condiciones similares a las últimas mencionadas). La diferencia en los resultados se debe probablemente al tipo de efluente, a la concentración inicial y en especial al pH de trabajo, ya que los experimentos aquí reportados se efectuaron 
a $\mathrm{pH}$ 2.80, en un efluente real y con una concentración inicial de tensoactivos (SAAM) de $8.24 \mathrm{mg} / \mathrm{L}$, mientras que Bandala et al. (2008) trabajaron en muestras sintéticas, sin ajuste de $\mathrm{pH}(\mathrm{pH}=5)$ y con una concentración inicial de dodecilsulfato de sodio de $1000 \mathrm{mg} / \mathrm{L}$.

Por otro lado, Isch (2016) alcanzó una reducción de tensoactivos mayor al $99 \%$ en un efluente sintético, con una concentración inicial de $1000 \mathrm{mg} / \mathrm{L}$, al cabo de $80 \mathrm{~min}$ mediante $60 \mathrm{mM}$ de $\mathrm{H}_{2} \mathrm{O}_{2}$ y 60 $\mathrm{mM}$ de $\mathrm{Fe}^{2+}$. En este estudio, a un tiempo semejante (90 min) y con una concentración de $\mathrm{Fe}^{2+}$ seis veces menor $\left(56.33 \mathrm{mM} \mathrm{H}_{2} \mathrm{O}_{2} ; 9.39 \mathrm{mM} \mathrm{Fe}^{2+}\right)$ se consiguió un $96 \%$ de remoción, lo cual es significativo si se considera también que por la naturaleza del efluente tratado existen compuestos que pueden generar competencia en el consumo de radicales hidroxilo (Rodríguez-Chueca et al. 2012).

Los datos presentados en la figura 6 demuestran que para todos los experimentos, la reacción Fenton convencional prácticamente se detuvo a los $120 \mathrm{~min}$, y en dicho tiempo se determinó, mediante una gráfica de medias (Fig. 7), que no existieron diferencias estadísticamente significativas entre las relaciones 1:9 y $1: 6$ de $\mathrm{Fe}^{2+}: \mathrm{H}_{2} \mathrm{O}_{2}$. Es decir, un incremento en la concentración de hierro no representó un aumento considerable en la remoción de tensoactivos, situación que ocurrió posiblemente por el consumo de por parte de los iones $\mathrm{Fe}^{2+}$ (Babuponnusami y Muthukumar 2014). Kos et al. (2010) incluso señalan que un exceso en las concentraciones de hierro afecta

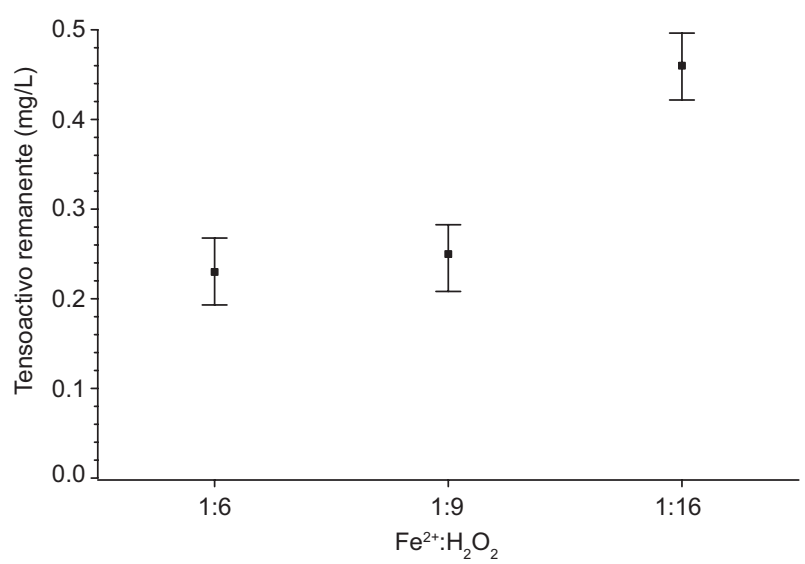

Fig. 7. Gráfica de medias $(95.0 \%$, prueba de diferencia mínima significativa de Fisher) de las concentraciones de sustancias activas al azul de metileno en el efluente de la planta de tratamiento de aguas residuales de Chaquibamba, tratado mediante un proceso Fenton convencional a diferentes relaciones molares $\mathrm{Fe}^{2+}: \mathrm{H}_{2} \mathrm{O}_{2}(\mathrm{pH}=2.80$, $\left.\left[\mathrm{H}_{2} \mathrm{O}_{2}\right]=56.33 \mathrm{mM}\right)$ de forma negativa a la remoción de contaminantes en aguas residuales textiles.

Es importante mencionar que al cabo de $120 \mathrm{~min}$ de tratamiento, la concentración de tensoactivos cumplió con lo estipulado en la legislación ecuatoriana y con lo recomendado por la USEPA para fuentes de agua destinadas al consumo público, independientemente de la relación molar utilizada (USEPA 1972, MAE 2015). Además, para todas las experimentaciones realizadas en esta etapa se observó una inactivación total de coliformes fecales después de 15 min de tratamiento.

En respuesta a los resultados expuestos en esta etapa, la relación molar 1:9 de $\mathrm{Fe}^{2+}: \mathrm{H}_{2} \mathrm{O}_{2}$ fue seleccionada para continuar con los ensayos previstos. Esta elección también evita la generación de una mayor cantidad de lodos (precipitados de hierro), los cuales presentaron una textura pastosa y una coloración pardo-rojiza durante la investigación realizada. La precipitación tuvo lugar al incrementar el $\mathrm{pH}$ de la solución hasta un valor de 12.0, acción realizada para descomponer el $\mathrm{H}_{2} \mathrm{O}_{2}$ residual.

\section{Proceso Fenton modificado con ácido cítrico}

En un proceso Fenton modificado con ácido cítrico se analizaron los efectos de la variación del pH y de la relación molar $\mathrm{Fe}^{2+}$ :citrato. En cada uno de los ensayos propuestos en esta etapa se alcanzó la inactivación total de coliformes fecales después de 15 min de tratamiento.

Por otro lado, la variación del porcentaje remanente de tensoactivos (SAAM) en el efluente de la PTAR de Chaquibamba, tratado mediante Fenton modificado se muestra en la figura 8. Del análisis estadístico correspondiente se determinó que el $\mathrm{pH}$ y relación molar $\mathrm{Fe}^{2+}$ : citrato presentaron efectos significativos durante el tratamiento y que existió una interacción entre las variables de diseño nombradas anteriormente.

En la figura 8 se muestra que al utilizar la relación $\mathrm{Fe}^{2+}$ :citrato de 1:1, la remoción de tensoactivos obtenida en los tratamientos fue mayor que aquella alcanzada al aplicar la relación 1:1.25; excepto al trabajar a $\mathrm{pH}$ del efluente $(\mathrm{pH}=7.84)$, cuyos resultados fueron contrarios. Una menor eficacia al utilizar la relación 1:1.25 se debió a que el citrato probablemente actuó como oxidante de los radicales hidroxilo e interfirió en la reacción de los tensoactivos con dichos radicales (Pignatello et al. 2006).

Al igual que en este trabajo, la relación molar $\mathrm{Fe}^{2+}$ :quelante $1: 1$ ha sido aplicada y sugerida, en el tratamiento de efluentes contaminados con tensoactivos (Isch 2016), así como en la degradación 


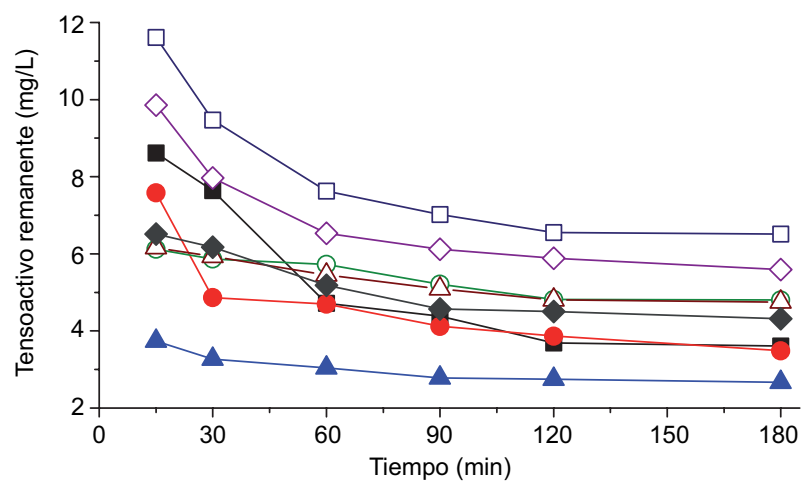

$-\mathrm{Fe}^{2+}:$ citrato $=1: 1 \mathrm{pH}=2.80-\bigcirc-\mathrm{Fe}^{2+}:$ citrato $=1: 1.25 \mathrm{pH}=2.80$
$-\mathrm{Fe}^{2+}:$ citrato $=1: 1 \mathrm{pH}=4.50-\triangle-\mathrm{Fe}^{2+}:$ citrato $=1: 1.25 \mathrm{pH}=4.50$
$-\mathrm{Fe}^{2+}:$ citrato $=1: 1 \mathrm{pH}=6.00--\mathrm{Fe}^{2+}:$ citrato $=1: 1.25 \mathrm{pH}=6.00$
$-\square-\mathrm{Fe}^{2+}:$ citrato $=1: 1 \mathrm{pH}=7.84--\mathrm{Fe}^{2+}:$ citrato $=1: 1.25 \mathrm{pH}=7.84$

Fig. 8. Variación del porcentaje remanente de sustancias activas al azul de metileno en el efluente de la planta de tratamiento de aguas residuales de Chaquibamba, tratado mediante Fenton modificado con ácido cítrico a diferentes valores de $\mathrm{pH}$ y relaciones molares $\mathrm{Fe}^{2+}$ : citrato $\left(\left[\mathrm{H}_{2} \mathrm{O}_{2}\right]\right.$ $=56.33 \mathrm{mM}$, relación molar $\mathrm{Fe}^{2+}: \mathrm{H}_{2} \mathrm{O}_{2}=1: 9$ )

de tricloroetileno (Lewis et al. 2009). No obstante, De Luca et al. (2014) encontraron que un exceso de agente quelante permitió un mayor porcentaje de complejamiento (quelante- $\mathrm{Fe}^{3+}$ ) y con ello alcanzó una mayor remoción de sulfametoxazol. La discrepancia existente se atribuiría al agente quelante evaluado, al contaminante tratado y al estado de oxidación en que se encontró el hierro.

Por otro lado, la remoción de tensoactivos fue mayor al incrementar el valor del $\mathrm{pH}$, excepto el $\mathrm{pH}$ del efluente (7.84), puesto que la remoción volvió a disminuir. Este comportamiento se puede atribuir a que un aumento del $\mathrm{pH}$ contribuye a la formación de especies como $\mathrm{Fe}\left(\mathrm{H}_{2} \mathrm{O}\right)_{4}(\mathrm{OH})_{2}$ que presentan mayor reactividad, es decir, aumentan la velocidad de reacción de descomposición del $\mathrm{H}_{2} \mathrm{O}_{2}$ (generación de ${ }^{\circ} \mathrm{OH}$ ) que describe la ecuación 1 (Pignatello et al. 2006, Lewis et al. 2009). La existencia de estas especies es posible debido a la presencia de citrato como agente quelante, el cual al formar complejos de hierro evita la presencia de especies formadas por el $\mathrm{Fe}^{3+}$ que son menos reactivas, y que a $\mathrm{pH}$ mayores a 3.00 tienden a coprecipitarse con los $\mathrm{Fe}^{2+}$ cuando ambos iones están presentes (Pignatello et al. 2006).

Es importante considerar que pese a iniciar el proceso con un complejo citrato- $\mathrm{Fe}^{2+}$ es probable la formación del complejo con el ion férrico y la coexistencia de ambos, lo cual pudo coadyuvar a la remoción de tensoactivos. Sin embargo, al incrementarse el pH a 7.84, el comportamiento mencionado cesa, tal vez porque la estabilidad del complejo citrato- $\mathrm{Fe}^{3+}$ disminuye; esto se refleja en el valor de la constante de disociación, la cual toma valores de 7.0 a 4.5 al cambiar el pH de 6.00 a 7.84 (Calderón 1997). Además, la posible formación de complejos con más de un núcleo de coordinación y con una actividad catalítica menor pudo incidir en la menor remoción de tensoactivos a $\mathrm{pH} 7.84$.

Para continuar con la siguiente etapa se realizó el análisis estadístico necesario y se determinó mediante una gráfica de interacción (Fig. 9) que los mejores resultados fueron obtenidos al aplicar una relación molar $\mathrm{Fe}^{2+}$ :citrato de $1: 1$ en un efluente acondicionado a un $\mathrm{pH}$ de 6 . En estas condiciones, se obtuvo una concentración de tensoactivos de $0.31 \mathrm{mg} / \mathrm{L}(\sim 96 \%$ remoción) en 15 min de tratamiento. La concentración citada es inferior al límite máximo establecido por las leyes ecuatorianas para descarga a un cuerpo de agua dulce, límite que se cita en el cuadro I.

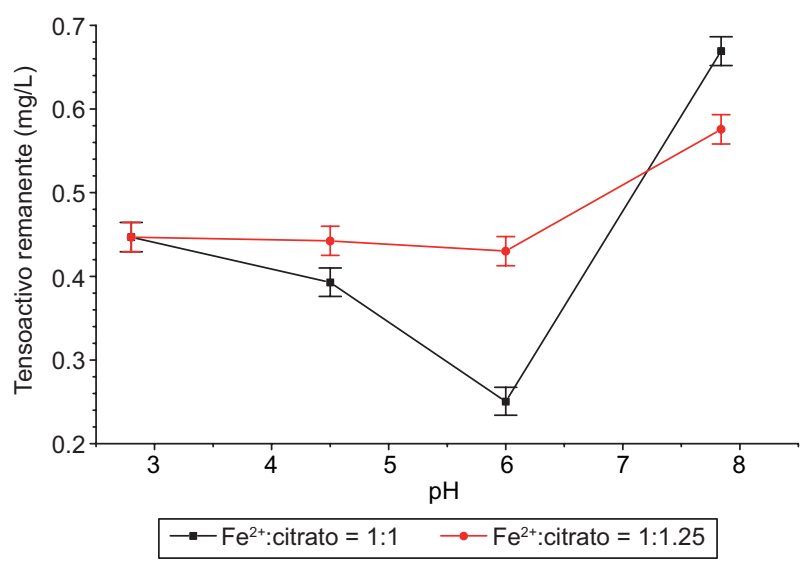

Fig. 9. Gráfica de interacción $(95.0 \%$, prueba de diferencia mínima significativa de Fisher) de las concentraciones de sustancias activas al azul de metileno en el efluente de la planta de tratamiento de aguas residuales de Chaquibamba, tratado mediante Fenton modificado con ácido cítrico a diferentes $\mathrm{pH}$ y relaciones molares $\mathrm{Fe}^{2+}$ :citrato $\left(\left[\mathrm{H}_{2} \mathrm{O}_{2}\right]=56.33 \mathrm{mM}\right.$, relación molar $\left.\mathrm{Fe}^{2+}: \mathrm{H}_{2} \mathrm{O}_{2}=1: 9\right)$

Hallazgos realizados por Lewis et al. (2009) muestran que debido a la quelación con citrato, la descomposición y degradación de tricloroetileno se efectuó a tasas menores que sin un quelato y que además se alcanzó un menor porcentaje de remoción de dicho contaminante. Los resultados aquí mostrados contrastan con lo citado dichos autores, porque tanto para el Fenton tradicional como para el 
modificado, en condiciones similares $(56.33 \mathrm{mM}$ $\mathrm{H}_{2} \mathrm{O}_{2}$ y Fe${ }^{2+}: \mathrm{H}_{2} \mathrm{O}_{2}=1: 9$ ), el porcentaje máximo de remoción de tensoactivos que se alcanzó fue de $97 \%$. Incluso, al evaluar la disminución de tensoactivos en $15 \mathrm{~min}$, el proceso Fenton modificado en las mejores condiciones permitió una mayor remoción $(96 \%)$ frente al correspondiente proceso Fenton tradicional (92\%). La discrepancia entre los resultados expuestos en este trabajo y los obtenidos por Lewis et al. (2009) puede deberse, en gran medida, a las características del contaminante tratado.

En cuanto al desempeño del citrato (ácido cítrico) como agente quelante, es posible mencionar que representa una alternativa viable frente a otros agentes que han sido objeto de estudio, tales como el ácido etilendiaminotetraacético (EDTA), oxalato, tartrato y succinato (Xue et al. 2009, Isch 2016), puesto que al aplicar un proceso Fenton modificado con citrato en un rango de $\mathrm{pH}$ de 2.80 a 7.84 se obtuvieron remociones mayores al $88 \%$ de tensoactivos (SAAM) e inactivaciones completas de coliformes fecales, en 15 min de tratamiento.

\section{Demanda química de oxígeno en las mejores con-} diciones del Fenton modificado

La última etapa de la investigación consistió en evaluar la reducción de la DQO. En el cuadro II se presenta el cambio en la concentración de tensoactivos, coliformes fecales y DQO en una nueva muestra compuesta tratada en las mejores condiciones del Fenton modificado con ácido cítrico. Es importante destacar que los valores iniciales de $\mathrm{pH}$, tensoactivos (SAAM) y coliformes fecales estuvieron dentro de los límites de control establecidos en la etapa de caracterización.

En esta experimentación final, en $15 \mathrm{~min}$ de tratamiento se alcanzó una disminución completa de coliformes fecales, un $97 \%$ de remoción de tensoactivos y un $89 \%$ de reducción de DQO.

Otras investigaciones (Rodríguez-Chueca et al. 2012) muestran que la materia orgánica y especies inorgánicas presentes en las aguas residuales domésticas actúan como eliminadores de los radicales hidroxilo, respecto a la inactivación de Escherichia coli y por ende disminuye la efectividad de la desinfección. En este trabajo, la carga presente de DQO no afectó la eficacia de los procesos Fenton estudiados, sin embargo, se puede inferir que valores iniciales de DQO más altos tienen una influencia negativa en la remoción de tensoactivos y la inactivación de Escherichia coli.

Los datos registrados en el cuadro II, así como los demás resultados expuestos en esta investigación, muestran el potencial de los procesos Fenton en el tratamiento de efluentes domésticos. Durante mucho tiempo se ha discutido respecto del mayor problema de los POA, que son los costos que conlleva su aplicación industrial, ya que los reactivos suelen ser caros (Esplugas et al. 2002, Asghar et al. 2015). Sin embargo, a pesar de los costos que pueden implicar estos procesos, se vislumbra que existirá la necesidad de aplicarlos al tratamiento de aguas residuales de tipo doméstico, por lo menos en casos particulares.

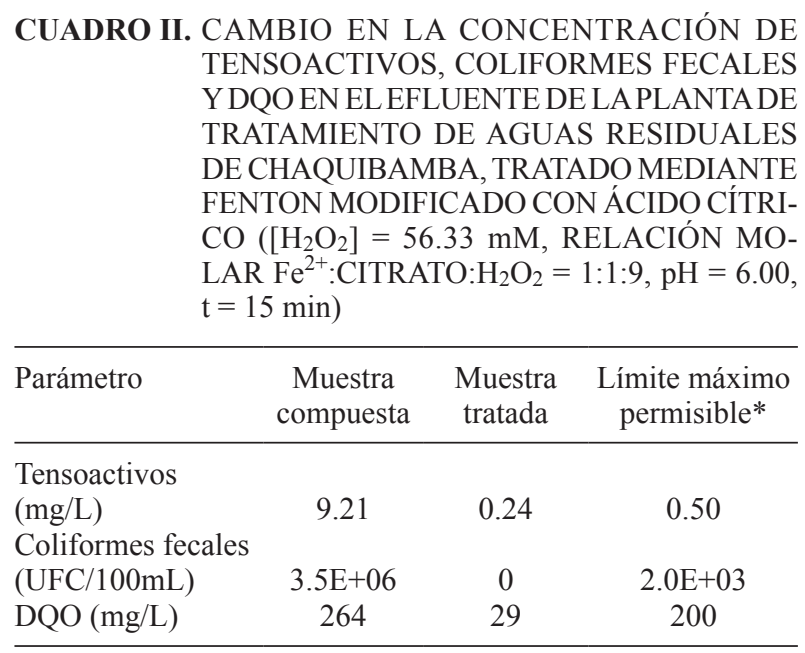

*MAE 2015, UFC: unidades formadoras de colonias

Se ha reconocido que el agente crucial para que bacterias de origen humano y animal tengan contacto con bacterias ambientales es el agua (Baquero et al. 2008). Por tanto, deberían realizarse grandes esfuerzos para eliminar totalmente las bacterias presentes en aguas residuales domésticas tratadas antes de su descarga a un cuerpo de agua receptor. Como dato, se presume que en la epidemia de cólera de 1992 en Latinoamérica, $V$. cholerae habría adquirido resistencia a antibióticos como resultado del contacto con bacterias resistentes seleccionadas debido al intensivo uso de antibióticos en la industria ecuatoriana de camarón (Cabello 2006), cuyas aguas entraron en contacto con bacterias ambientales durante muchos años. Por tanto, es lógico pensar que instituciones que utilizan antibióticos intensivamente, como centros de salud y asilos de ancianos, tendrían que llevar a cabo un tratamiento previo de sus aguas antes de la descarga al alcantarillado doméstico. Los únicos sistemas de tratamiento suficientemente compactos para lograrlo serían los basados en POA. Queda 
claro que se requiere realizar más investigaciones para lograr mejoras considerables en los costos y la eficiencia de estos procesos.

\section{CONCLUSIONES}

La caracterización del efluente de la PTAR del barrio Chaquibamba evidenció que los parámetros tensoactivos, coliformes fecales y DQO incumplieron con los límites permisibles estipulados en la legislación ecuatoriana vigente, lo que obligó a realizar un tratamiento complementario.

Las mejores condiciones para disminuir tensoactivos y coliformes fecales en aguas residuales domésticas mediante un proceso Fenton convencional fueron $56.33 \mathrm{mM}$ de $\mathrm{H}_{2} \mathrm{O}_{2}$, una relación molar $\mathrm{Fe}^{2+}: \mathrm{H}_{2} \mathrm{O}_{2}$ de $1: 9$ y agitación continua a $130 \mathrm{rpm}$, con lo cual se alcanzó una disminución completa de coliformes fecales en 15 min y una concentración de tensoactivos de $0.25 \mathrm{mg} / \mathrm{L}$ después de $120 \mathrm{~min}$.

Las mejores condiciones para disminuir los parámetros de estudio mediante un proceso Fenton modificado con ácido cítrico fueron $56.33 \mathrm{mM}$ de $\mathrm{H}_{2} \mathrm{O}_{2}$, una relación molar $\mathrm{Fe}^{2+}$ : citrato: $\mathrm{H}_{2} \mathrm{O}_{2}$ de $1: 1: 9$, un $\mathrm{pH}$ de 6.00 y agitación continua a $130 \mathrm{rpm}$, con lo cual se alcanzó la inactivación completa de coliformes fecales y una concentración de tensoactivos de $0.31 \mathrm{mg} / \mathrm{L}$ en $15 \mathrm{~min}$ de tratamiento. La remoción de tensoactivos mediante Fenton modificado con ácido cítrico se efectuó en un menor tiempo que la remoción obtenida mediante un proceso Fenton tradicional.

La aplicación de las mejores condiciones para disminuir tensoactivos y coliformes fecales en aguas residuales domésticas mediante un proceso Fenton modificado, permitió reducir la DQO de $264 \mathrm{mg} / \mathrm{L} \mathrm{a}$ $29 \mathrm{mg} / \mathrm{L}$ en $15 \mathrm{~min}$.

Los procesos Fenton convencional y modificado permitieron que los parámetros tensoactivos y coliformes fecales alcanzaran valores por debajo de los límites establecidos en la normativa ecuatoriana para descarga de efluentes a un cuerpo de agua dulce, y que cumplieran con las recomendaciones establecidas por la USEPA para fuentes de agua destinadas al consumo público.

La incorporación de un proceso Fenton modificado como tratamiento complementario de efluentes domésticos tiene el potencial de reducir tensoactivos, inactivar coliformes fecales y disminuir la DQO. Además, dada la capacidad de estos POA para la remoción de coliformes fecales, deberían considerarse como parte de las estrategias mundiales en la lucha contra las bacterias resistentes a los antibióticos.

\section{REFERENCIAS}

Abbot J. y Brown D.G. (1990). Kinetics of iron-catalyzed decomposition of hydrogen peroxide in alkaline solution. Int. J. Chem. Kinet. 22 (9), 963-974.

DOI: 10.1002/kin.550220907

Al-Jassim N., Ansari M.I., Harb M. y Hong P.-Y. (2015). Removal of bacterial contaminants and antibiotic resistance genes by conventional wastewater treatment processes in Saudi Arabia: Is the treated wastewater safe to reuse for agricultural irrigation? Water Res. 73, 277-290. DOI: 10.1016/j.watres.2015.01.036

APHA/AWWA/WEF (2012). Standard methods for the examination of water and wastewater. 22a ed. American Public Health Association/American Water Works Association/Water Environment Federation, Washington D.C., EUA, 1496 pp.

Asghar A., Abdul-Raman A.A. y Daud W.M.A.W. (2015). Advanced oxidation processes for in-situ production of hydrogen peroxide/hydroxyl radical for textile wastewater treatment: A review. J. Cleaner Prod. 87, 826-838. DOI: 10.1016/j.jclepro.2014.09.010

Babuponnusami A. y Muthukumar K. (2014). A review on Fenton and improvements to the Fenton process for wastewater treatment. J. Environ. Chem. Eng. 2 (1), 557-572. DOI: 10.1016/j.jece.2013.10.011

Bandala E.R., Peláez M.A., Salgado M.J. y Torres L. (2008). Degradation of sodium dodecyl sulphate in water using solar driven Fenton-like advanced oxidation processes. J. Hazard. Mater. 151 (2-3), 578-584. DOI: 10.1016/j.jhazmat.2007.06.025

Baquero F., Martínez, J.-L. y Cantón R. (2008). Antibiotics and antibiotic resistance in water environments. Curr. Opin. Biotechnol. 19 (3), 260-265.

DOI: $10.1016 /$ j.copbio.2008.05.006

Cabello F.C. (2006). Heavy use of prophylactic antibiotics in aquaculture: A growing problem for human and animal health and for the environment. Environ. Microbiol. 8 (7), 1137-1144.

DOI: 10.1111/j.1462-2920.2006.01054.x

Cabiscol E., Tamarit J. y Ros J. (2000). Oxidative stress in bacteria and protein damage by reactive oxygen species. Int. Microbiol. 3 (1), 3-8.

Calderón F. (1997). Todo sobre los quelatos. Dr. Calderón laboratorios, Bogotá, Colombia, 13 pp.

Chitra S., Paramasivan K., Shanmugamani A.G., Rao S.V.S. y Paul B. (2014). Advanced oxidation processes for the treatment of surfactant wastes. J. Chem. Eng. Chem. Res. 1 (3), 163-173.

De Luca A., Dantas R.F. y Esplugas S. (2014). Assessment of iron chelates efficiency for photo-Fenton at neutral pH. Water Res. 61, 232-242.

DOI: 10.1016/j.watres.2014.05.033 
Edberg S.C., Rice E.W., Karlin R.J. y Allen M.J. (2000). Escherichia coli: The best biological drinking water indicator for public health protection. Soc. Appl. Microbiol. Symp. Ser. 88(29), 106S-116S.

DOI: 10.1111/j.1365-2672.2000.tb05338.x.

Esplugas S., Giménez J., Contreras S., Pascual E. y Rodríguez M. (2002). Comparison of different advanced oxidation processes for phenol degradation. Water Res. 36 (4), 1034-1042.

DOI: 10.1016/S0043-1354(01)00301-3

Giannakis S., Melvin-Le T.-T., Entenza J.M. y Pulgarin C. (2018). Solar photo-Fenton disinfection of 11 antibiotic-resistant bacteria (ARB) and elimination of representative AR genes. Evidence that antibiotic resistance does not imply resistance to oxidative treatment. Water Res. 143, 334-345.

DOI 10.1016/j.watres.2018.06.062

Gogate P.R. y Pandit A.B. (2004). A review of imperative technologies for wastewater treatment I: oxidation technologies at ambient conditions. Adv. Environ. Res. 8 (3-4), 501-551. DOI: $10.1016 / \mathrm{S} 1093-0191(03) 00032-7$

Goi A. y Trapido M. (2002). Hydrogen peroxide photolysis, Fenton reagent and photo-Fenton for the degradation of nitrophenols: A comparative study. Chemosphere 46 (6), 913-922.

DOI: 10.1016/S0045-6535(01)00203-X

Heinze S., Chen Y., el-Nahhal Y., Hadar Y., Jung R., Safi J., Safi M., Tarchitzky J. y Marschner B. (2014). Small scale stratification of microbial activity parameters in mediterranean soils under freshwater and treated wastewater irrigation. Soil Biol. Biochem. 70, 193-204. DOI: 10.1016/j.soilbio.2013.12.023

Hernández A. (2012). Evaluación de fotocatálisis heterogénea con $\mathrm{TiO}_{2}$ y proceso Fenton para el control de Salmonella spp en aguas generadas en plantas de beneficio avícola. Tesis de Maestría. Facultad de Ciencias, Pontificia Universidad Javeriana, Bogotá, Colombia, $121 \mathrm{pp}$.

Isch T. (2016). Estudio de un sistema para el tratamiento de efluentes contaminados con compuestos surfactantes por un método Fenton y Fenton modificado con un agente quelante (EDTA). Tesis de Licenciatura. Facultad de Ingeniería Química y Agroindustria, Escuela Politécnica Nacional, Quito, Ecuador, 104 pp.

Ivanković T. y Hrenović J. (2010). Surfactants in the environment. Arh. Hig. Rada Toksikol. 61 (1), 95-110. DOI: 10.2478/10004-1254-61-2010-1943

Kitis M., Adams C.D. y Daigger G.T. (1999). The effects of Fenton's reagent pretreatment on the biodegradability of nonionic surfactants. Water Res. 33 (11), 2561-2568.

DOI: 10.1016/S0043-1354(98)00476-X
Klamerth N., Malato S., Agüera A. y Fernández-Alba A. (2013). Photo-Fenton modified photo-Fenton at neutral $\mathrm{pH}$ for the treatment of emerging contaminants in wastewater treatment plant effluents: a comparison. Water Res. 47 (2), 833-840. DOI: $10.1016 /$ j.watres.2012.11. 008

Kos L., Michalska K. y Perkowski J. (2010). Textile wastewater treatment by the Fenton method. Fibres Text. East. Eur. 18 (4), 105-109.

Lee R.J. y Saylor J.R. (2010). The effect of a surfactant monolayer on oxygen transfer across an air/water interface during mixed convection. Int. J. Heat Mass Transfer 53 (17-18), 3405-3413.

DOI:10.1016/j.ijheatmasstransfer.2010.03.037

Lewis S., Lynch A., Bachas L., Hampson S., Ormsbee L. y Bhattacharyya D. (2009). Chelate-modified Fenton reaction for the degradation of trichloroethylene in aqueous and two-phase systems. Environ. Eng. Sci. 26 (4), 849-859. DOI: 10.1089/ees.2008. 0277

Mara D. (2003). Domestic wastewater treatment in developing countries. Earthscan, Londres, Reino Unido, $293 \mathrm{pp}$.

MAE (2015). Acuerdo Ministerial núm. 097-A: Refórmese el texto unificado de legislación secundaria. Ministerio del Ambiente del Ecuador. Registro Oficial, 4 de noviembre.

NMKL (2015). NMKL Newsletter 90. Nordisk Metodikkomité for Næringsmidler [en línea]. http://www.nmkl. org/dokumenter/nytt/90-eng.pdf 27/04/2016

Oromí-Durich J. (2000). Resistencia bacteriana a los antibióticos. Medicina Integral 36 (10), 367-370.

Pepper I.L., Gerba C.P. y Gentry T.J. (2014). Environmental microbiology. 3a ed. Academic Press, San Diego, EUA, 728 pp. DOI: 10.1016/B978-0-12-394626-3

Pignatello J.J., Oliveros E. y MacKay A. (2006). Advanced oxidation processes for organic contaminant destruction based on the Fenton reaction and related chemistry. Crit. Rev. Environ. Sci. Technol. 36 (1), 1-84. DOI: $10.1080 / 10643380500326564$

Ramin N., Mahmood A., Hasan A., Alireza M., Kazem N., Reza N. y Maryam G. (2012). Comparative study of Fenton's reagent performance in disinfection of raw wastewater and activated sludge effluent. Desalin. Water Treat. 37 (1-3), 108-113. DOI:10.1080/19443994.2012. 661261

Rodríguez-Chueca J., Mosteo R., Ormad M.P. y Ovelleiro J.L. (2012). Factorial experimental design applied to Escherichia coli disinfection by Fenton and photoFenton processes. Sol. Energy 86 (11), 3260-3267. DOI: 10.1016/j.solener.2012.08.015

Rosen M.J. y Kunjappu J.T. (2012). Surfactants and interfacial phenomena. 4a ed. John Wiley and Sons, Nueva Jersey, EUA, 616 pp. 
Selvakumar A., Tuccillo M.E., Muthukrishnan S. y Ray A.B. (2009). Use of Fenton's reagent as a desinfectant. Remediation Journal 19 (2), 135-142.

DOI: $10.1002 /$ rem.20208

Seol Y. y Javandel I. (2008). Citric acid-modified Fenton's reaction for the oxidation of chlorinated ethylenes in soil solution systems. Chemosphere 72 (4), 537-542. DOI: 10.1016/j.chemosphere.2008.03.052

USEPA (1972). Water Quality Criteria 1972. Informe. United States Environmental Protection Agency, Washington D.C., EUA, 594 pp.

Voloshenko-Rossin A., Gasser G., Cohen K., Gun J., Cumbal-Flores L., Parra-Morales W., Sarabia F., Ojeda F. y Lev O. (2015). Emerging pollutants in the Esmeraldas watershed in Ecuador: Discharge and attenuation of emerging organic pollutants along the San PedroGuayllabamba-Esmeraldas rivers. Environ. Sci.-Proc. Imp. 17 (1), 41-53. DOI: 10.1039/c4em00394b
Xue X., Hanna K., Despas C., Wu F. y Deng N. (2009). Effect of chelating agent on the oxidation rate of PCP in the magnetite $/ \mathrm{H}_{2} \mathrm{O}_{2}$ system at neutral $\mathrm{pH}$. J. Mol. Catal. A-Chem. 311 (1-2), 29-35. DOI: $10.1016 /$ j.molcata.2009.06.016

Yaya-Beas R.-E., Cadillo-La-Torre E.-A., Kujawa-Roeleveld K., van Lier J.B. y Zeeman G. (2016). Presence of helminth eggs in domestic wastewater and its removal at low temperature UASB reactors in peruvian highlands. Water Res. 90, 286-293.

DOI: $10.1016 /$ j.watres.2015.11.023 